\title{
I. Umfang und Beschaffenheit des Materials.
}

Uber die Abgrenzung des handschriftlichen Nachlasses von den ersten beiden Abtheilungen der Werke und des Briefwechsels hat der Leiter der Ausgabe im Vorwort zum I. Bande (S. X-XII) berichtet.

Für den grössten Theil des Materials konnten Kants Aufzeichnungen selbst zugrunde gelegt werden. Im Manuscript nicht auffindbar, beziehungsweise nicht zugänglich waren nur die von Rink herausgegebenen Vorarbeiten zur Beantwortung der Preisfrage betreffend die Fortschritte der deutschen Metaphysik seit Leibniz und Wolff, die sieben kleinen Aufsätze von 1788-91, sowie einige andere der von Schubert und Reicke aus Kants Nachlass veröffentlichten Blätter, bei deren jedem diese Thatsache Erwähnung finden wird.

Im Ubrigen gestatteten Privatleute wie die meisten Bibliotheken in seltnem Entgegenkommen und Vertrauen häusliche Benutzung ibrer Kant-Manuscripte, grösstentheils für lange Jahre. Diesem Umstand ist es vor allem zuzuschreiben, dass die Datirung der einzelnen Aufzeichnungen eine weit genauere hat werden können, als nach den friheren Veröffentlichungen zu erwarten war. Unerlässliche Bedingung für den Erfolg war, dass der Herausgeber in den Handschriften ganz heimisch wurde: dass er immer wieder, bei verschiedener Beleuchtung, die einzelnen Blätter auf Tinte und Schrift vergleichen, an schwer zu entziffernden Worten sich versuchen und nicht gebunden an bestimmte, oft kärglich bemessene Bibliothekszeiten - in jedem Angenblick zu seinen Problemen zurückkehren konnte, um neu auftauchende Lösungsmöglichkeiten sofort $\mathrm{zu}$ verfolgen; so hat oft die Gunst einer Stunde Licht gebracht in ein Dunkel, das wochenlange Arbeit umsonst zu erhellen bemüht gewesen war. 
Der letzte Band dieser Abtheilung wird gelegentlich der Beschreibung der einzelnen Manuscripte auch deren Besitzer angeben und an besonderer Stelle die Namen derjenigen verzeichnen, die ihre Schätze in den Dienst der Ausgabe gestellt haben und die dafür des bleibenden Dankes der Wissenschaft sicher sein können.

Nur wenige Aufzeichnungen finden sich in dieser Abtheilung, die bei der Niederschrift von Kant für die Öffentlichkeit (wie die ursprüngliche Einleitung zur „Kritik der Urtheilskraft") oder wenigstens zur directen Mittheilung an einzelne Personen (wie die sieben kleinen Aufsätze für Kiesewetter aus den Jahren 1788-91) bestimmt waren.

Alles andere hat er nur zu seinem Privatgebrauch aufgezeichnet: sei es als Material für seine Vorlesungen, sei es als Vorarbeit oder Entwurf zu seinen Druckwerken, sei es bloss in der Absicht, seine Gedanken zu fixiren und zu klären.

Diese Manuscripte für den eigenen Gebrauch zerfallen, äusserlich betrachtet, in zwei Klassen: einzelne Blätter und Handexemplare eigner oder fremder Schriften.

Die erste Art hat R. Reicke als „Lose Blätter aus Kants Nachlass" bezeichnet. Mit Recht. Denn nur selten besteht zwischen ihnen eine erkennbare äussere Verbindung, noch seltener einen sie sich durch Numerirung zu grösseren Zusammenhängen in geordneten Lagen. Ihr Format ist sehr verschiedenartig: vom Foliobogen and Quartblatt bis herab zu kleinen Papierschnitzeln sind alle Grössen vertreten, bald regelmässig beschnitten, bald unregelmässig abgerissen. Anch auf den einzelnen Zetteln fehlt es häufig an innerlicher Einheit; sehr verschiedenartige Gegenstände werden oft nach oder durch einander auf ein em Blatt behandelt, ein wagerechter Strich von zwei, drei Centimeter Länge an der linken Seite des Blattes dient dann gewöhnlich als Scheidewand zwischen je zwei Bemerkungen.

Es war Kants Gewohnheit, auf solchen Zetteln zu vermerken, was ihn in wissenschaftlichen Dingen interessirte, beschäftigte, quälte, aber auch sonst allerlei, was ihm gerade durch den Kopf ging und seinem Gedächtniss ohne solche Nachhülfe leicht hätte entschwinden können. So finden wir denn über die losen Blätter in buntem Wechsel grössere und kleinere Entwürfe zerstrent, unermüdlich wiederholte Versuche, einem Problem von hier oder von dort her beizukommen, fur 
die Darstellang einer neu erarbeiteten Erkenntniss die passendsten Ausdrücke, die geeignetste Gedankenfolge zu finden, Vorarbeiten zu seinen Veröffentlichungen aus den verschiedensten Stadien (von rohen Skizzen bis zur Reinschrift, von der sich der Druck nur noch durch Kleinigkeiten unterscheidet), Material für seine Vorlesungen (kurze thatsächliche Notizen zur Unterstützung des Gedächtnisses, skizzenhafte Entwürfe über grössere Gebiete, zum Theil in Telegrammstil verfasst, zusammenhängende Ausarbeitungen, mit einer Sorgfalt behandelt, als wären sie für die Öffentlichkeit bestimmt), ferner literarische Notizen, Excerpte, aber auch Rechnungen und auf Haushalt und sonstige Privatangelegenheiten bezügliche Bemerkungen. Blätter privaten Inhalts sind sehr häufig in den letzten Lebensjahren Kants, in der Zeit seiner Alters- und Gedächtnisschwäche; aus ihnen wird nur das zum Abdruck gebracht, was wissenschaftlichen Charakter trägt oder dazu dient, dies Letztere chronologisch zu bestimmen.

Von grosser Wichtigkeit ist, dass Kant zu seinen Aufzeichnungen gern allerhand an ihn gesandte Schreiben ganz oder stückweise benutzte. Oft sind auf ihnen Jahr und Datum noch erhalten, und dann ist es möglich, auch Kants Bemerkungen ziemlich genau zu datiren. Denn er pflegte diese Schriftstücke bald (einige Wochen, Monate) nach Eingang zu benutzen. Das lässt sich für eine Reihe von Briefen und Brieffragmenten, auf denen sich Vorarbeiten zu Druckwerken befinden, wo also ein terminus a quo und ein terminus ad quem gegeben sind, mit Sicherheit feststellen.

Die Blätter (zum weitaus grössten Theil im Besitz der Königlichen und Universitäts-Bibliothek zu Königsberg) vertheilen sich auf einen Zeitraum von rund 50 Jahren, die meisten aber entstammen dem letzten Jahrzehnt des 18. Jahrhunderts. Die für Kants Entwicklung so wichtigen $60 \mathrm{er}$ und $70 \mathrm{er}$ Jahre sind nur wenig vertreten, die 70 er jedoch mit um so wertvolleren Stücken.

Einen Theil der Königsberger Papiere hat R. Reicke in den drei Heften der "Losen Blätter aus Kants Nachlass" veröffentlicht, die zuerst in der ,Altpreussischen Monatsschrift" erschienen, dann selbständig 1889, 1895, 1898.

Von der zweiten Classe von Manuscripten: Handexemplaren Kants, in die er seine Bemerkungen eingetragen hat, liegen neun vor. 
Drei davon sind Schriften Kants:

1) ein mit Octavblättern durchschossenes Exemplar der ,Beobachtungen über das Geftahl des Schönen and Erhabenen" (1764),

2) ein Exemplar der „Kritik der reinen Vernunft" (1781),

3) ein Exemplar der „Kritik der praktischen Vernunft" (1788).

Sodann:

4) George Friedrich Meier: Auszug aus der Vernunftlehre. (Mit Quartblättern durchschossen.) Halle. 1752.

5) Alexander Gottlieb Baumgarten: Metaphysica. Editio IIII. (Mit Octavblättern durchschossen.) Halae Magdeburgicae. 1757.

6) Derselbe: Initia philosophiae practicae primae. Halae Magdeburgicae. 1760.

7) Gottfr. Achenwall: Juris naturalis pars posterior complectens jus familiae, jus publicum et jus gentium. Editio quinta emendatior. Gottingae. 1763.

8) Johann August Eberhard: Vorbereitung zur natürlichen Theologie zum Gebrauch akademischer Vorlesungen. (Mit Quartblättern durchschossen.) Halle. 1781.

9) Georg Christoph Lichtenberg: Vermischte Schriften, hrsgg. von Ludwig Christian Lichtenberg und Friedrich Kries. Bd. II. (Mit Octavblättern durchschossen.) Göttingen. 1801.

Die Nrn. 1, 2, 6-8 gehören der Königlichen und UniversitätsBibliothek zu Königsberg, Nr. 3 der Hallenser Universität, Nr. 4 und 5 der Dorpater Universitätsbibliothek, Nr. 9 Herrn Verlagsbuchhändler D. Minden in Dresden-Blasewitz.

Nr. 3 und 9 enthalten nur einige wenige Bemerkungen. Am wichtigsten sind die Nrn. 1, 4-7: die Aufzeichnungen in ihnen entstammen zum grösseren Theil der Zeit des Werdens vor 1781. Die reichste Ausbeute gewährt das Exemplar von Baumgartens „Metaphysica", und mehr als die Hälfte aller Reflexionen fällt hier in die Jahre des grossen Schweigens, etwa in das Jahrzehnt von 1769-1779. Benno Erdmann hat aus dem letzteren Werk eine Auswahl unter dem Titel : Reflexionen Kants zur kritischen Philosophie veröffentlicht (Bd. I. Heft 1: Reflexionen zur Anthropologie. 1882. Bd. II: Reflexionen zur Kritik der reinen Vernunft. 1884). 
Dem Handexemplar der „Beobachtungen" sind die Fragmente aus Kants Nachlass entnommen, die Schubert in Bd.XI Abth. 1 seiner Ansgabe (S. 221-260) in äusserst nachlässiger Weise, vielfach entstellt, mittheilte. Diese Bemerkungen zu den „Beobachtungen" waren vielleicht zunächst als Nachträge zu der Schrift gedacht, die in einer zweiten Auflage verwerthet werden sollten. Allmählich ist aber das Handexemplar zu einer allgemeinen Materialiensammlung geworden, wie die Reflexionen physikalischen und naturrechtlichen Inhalts beweisen, die sich in ihm finden.

Die Aufzeichnungen in dem Handexemplar der „Kritik der reinen Vernunft" dienten der Vorbereitung einer Neuauflage. B. Erdmann hat sie 1881 als „Nachträge zu Kants Kritik der reinen Vernunft" herausgegeben.

Die wenigen Bemerkungen im II. Bd. von Lichtenbergs ,,Vermischten Schriften" sind Gedanken, zu denen die Lectüre Kant anregte.

Die Compendien von Meier, Baumgarten, Achenwall, Eberhard dienten ihm als Grundlage für seine Vorlesungen. Der Gebrauch derartiger Lehrbücher war bekanntlich damals auf deutschen Universitäten allgemein und wurde den Königsberger Professoren durch ein Rescript des Ministers v. Zedlitz vom 16. Oktober 1778 noch besonders eingeschärtt. „Das schlechteste Compendium", hieß es hier, ,ist gewiss besser als keines, und die Professores mögen, wenn sie so viel Weisheit besitzen, ihren Autorem verbessern, so viel sie können, aber das Lesen über Dictata muß schlechterdings abgeschafft werden." Für Kant war die Angabe der Compendien bei Ankündigung seiner Vorlesungen mehr als eine leere Formel. Auch in den 80 er und 90 er Jahren schloss er sich noch immer wenigstens äusserlich an seinen „Autor" an, häufiger freilich widersprechend als zustimmend.

Kants Aufzeichnungen in diesen Compendien beziehen sich, in der früheren Zeit sämmtlich, später immerhin in ibrer grossen Mehrzahl, auf die Paragraphen der Lehrbücher und die in ihnen behandelten Probleme, dienten also als Material für seine Vorlesungen und wurden vermuthlich zu einem grossen Theil unmittelbar vor oder nach ihnen niedergeschrieben, sei es zur Vorbereitung, sei es als Resultat: als eine im freien Vortrag (IX 4, XII 387) erarbeitete Klärung und Bereicherung der Gedanken. 
Eine Ausnahme von der Regel machen nur gewisse Parthien in Baumgartens „Metaphysica": so vor allem 53 eng beschriebene Seiten im Anfang, vor Beginn des eigentlichen Textes (Vorsatzblätter, Titelblatt, die drei Praefationes und die Synopsis umfassend), in der Psychologia empirica die Durchschussblätter $215^{\prime}-219^{\prime}, 228^{\prime}-249^{\prime}$, soweit sie ästhetischen Inhalts sind, in der Psychologia rationalis die Textseiten und Durchschussblätter 293-313, 320-325, soweit sie anthropologische Bemerkungen enthalten, und von S. $403^{\prime}$ an fast der ganze Rest der Aufzeichnungen. Viele Reflexionen auf diesen Seiten stehn mit den Paragraphen des Lehrbuchs und dem möglichen Inhalt des Collegs auch nicht im entferntesten Zusammenhang; anderswo sind der Bemerkungen aus einer und derselben Zeit über ein und dasselbe Thema so viele, dass ihre Masse in keinem Verhältniss steht zu der Zeit, die im Colleg darauf hätte verwandt werden können; dann wieder fällt der bunte Wechsel auf, in dem die Gegenstände auf einander folgen; oder die häufige Wiederkehr derselben Gedanken, in verschiedenen Ausdrücken und von verschiedenen Ausgangspunkten her dargestellt, beweist, dass es Kant nicht darum zu thun war, durch sie zu lehren, sondern selbst aus ihnen zu lernen, d. h. durch wiederholte Niederschrift Klärung schwieriger Probleme zu erringen. In solchen Fällen - aber auch nur in ihnen! - haben die Seiten des Handexemplars als allgemeine Materialiensammlung, als ,wissenschaftliches Tagebuch" (Erdmann: Reflexionen I 30) gedient. Zum allergrössten Theil entstammen die betreffenden Bemerkungen dem Jahrzehnt intensivster Arbeit, in dem das System der kritischen Philosophie im Geist ihres Schöpfers allmählich concrescirte: den Jahren 1769-79.

Kants Aufzeichnungen sind über die Compendien sehr ungleich vertheilt. Viele Seiten, ja! oft ganze Reihen von Seiten, sind unbeschrieben. Auf anderen drängen sich die Bemerkungen um so mehr, vor allem za Beginn neuer Abschnitte oder wo Probleme behandelt werden, die Kant besonders lebhaft beschäftigten. Häufig sind dann nicht nur die Durchschussblätter und die Ränder der Textseiten ganz gefüllt: auch zwischen dem Text und zwischen den Zeilen der älteren Reflexionen stehen noch Bemerkungen, und selbst die kleinsten freien Plätzchen hatKant (namentlich in den $80 \mathrm{er} \mathrm{Jahren)} \mathrm{nicht} \mathrm{verschmäht,}$ 
um, wenn alles andere voll war, auf ihnen noch einige Reflexionen nothdürftig nnterzubringen, die dann freilich in zwei, drei, vier Stücke getrennt und durch verschieden gestaltete Fortsetzungszeichen mit einander verbunden werden mussten. So bieten manche Seiten ein sehr buntes Bild, und Kants Gedächtniss und scharfes Auge sind zu bewandern, wenn er sich in den Vorlesungen der $80 \mathrm{er}$ und $90 \mathrm{er}$ Jahre in dem beängstigenden Wirrwarr solcher Blätter noch zurechtfinden konnte.

Die Bedentung der Aufzeichnungen ist eine sehr angleiche. Manches scheint absolut werthlos. Wäre das nicht besser fortgeblieben? Was geht es die hentige und die künftige Philosophie an, ob und wie Kant seinen Autor inhaltlich oder stilistisch in dieser oder jener Kleinigkeit verbesserte? Und welches Interesse können wir an missglückten Versuchen von Gedankenformulirungen haben, wenn ihr schliessliches Resultat in Kants Werken vorliegt? Vor allem an Versuchen, die sich so überoft wiederholen, wie die in den Vorarbeiten zu den „metaphysischen Anfangsgründen der Rechtslehre"?

Ausserdem: es kann keinem Zweifel unterliegen, dass eine Veroffentlichung des ganzen handschriftlichen Nachlasses den Intentionen Kants nicht entspricht. Darauf zwar ist kein Gewicht zu legen, dass ein Testamentsentwurf aus dem Jahr 1791 (auf der Königliehen und Universitäts-Bibliotkek zu Königsberg, Nr. 29 im Convolut M der Kant-Blätter) dem Magister und Subinspector Gensichen Kants sämmtliche Bücher und Manuseripte vermacht mit folgender Clausel, die auch in das (wie es scheint, verloren gegangene) Testament vom 29. Angust 1791 übergegangen sein dürfte: „Zugleich ersucheich gedachten Herren Magister alle meine litterärische Papiere worunter ich auch die von mir zu meinen Vorlesungen gebrauchte und hăufig und für jeden andern unleserlich beschriebene Handbücher [übergeschrieben: für meine Vorlesungen] verstehe nachdem er sie nach seinem Belieben durchgesehen hat zu vernichten." Diese Clausel ist später nicht mehr nach Kants Sinne gewesen. Denn in einem Testamentsentwurf rom Jahr 1798 (auf der Rückseite des Blattes M 29) heißt es: „Meinem Freund Herren Professor Gensichen ver- 
mache ich ... meinen nicht sehr erheblichen ganzen Büchervorrath sammt meinen Manuscripten über sie nach Belieben doch nicht durch offentliche Auction unter meinem Nahmen zu verfügen." Und das Testament selbst vom 26. Februar 1798, durch welches das frühere vom Jahre 1791 aufgehoben wird, läßt auch das Auctionsverbot fallen, spricht freilich nicht von Manuseripten, sondern nur von dem, ganzen Büchervorrath" (XII 410); doch waren darin die Vorlesungscompendien selbstverständlich inbegriffen. Im Jahr 1799 sodann gingen diese letzteren, zusammen mit andern Manuseripten, in die Hände Jäsches und Rinks äber, denen von Seiten Kants der Auftrag ward, auf Grund jener Materialien Handbücher der einzelnen Wissenschaften, etwa nach Art seiner Anthropologie, zusammenzustellen (IX 3-4, 153-5, XII 398). Eine Veröffentlichung des ganzen in den Compendien enthaltenen Stoffes hätte Kant sich zweifelsohne - auch für die Zeit nach seinem Tode - verbeten, ebenso wie eine Edition der meisten seiner übrigen Manuscripte, vor allem auch der Vorarbeiten zu älteren Werken.

In demselben Sinn würde sich in gleicher Lage jeder Schriftsteller entscheiden, dem nicht Eitelkeit und Selbstüberschätzung die Augen völlig geblendet haben. Und der Durchschnittsmensch könnte auch verlangen, gehört za werden und seinen Willen durchzusetzen. Nicht so die bahnbrechenden Geister: sie werden mit anderen Maasstäben gemessen, und darum munssen sie sich gefallen lassen, dass auch andere Forderungen ibnen gegenuber geltend gemacht werden. Die Menschheit hat ein Recht an das, was sie sind, und an das, was sie schaffen. Und darum dürfen nicht sie, sondern erst die $\mathrm{Nachwelt}$ kann entscheiden, was von ihrer geistigen Arbeit werth ist, der Znkanft aufbewahrt und der Öffentlichkeit zugänglich gemacht zu werden.

Indem also die Ausgabe scheinbar pietätlos verfăhrt, lăsst sie sich in Wirklichkeit von echtester Pietät leiten. Sie zieht nur die Consequenz aus der Erkenntniss, dass Kant ein Platz in der ersten Reihe der grossen wissenschaftlichen Genies gebührt. Genies aber sind selten, nnd noch seltner sind die Fälle, wo es uns vergönnt ist, so tief in die Werkstatt ihres Geistes, in das Keimen und Wachsen ihrer Gedanken za blicken, wie der Kantische Nachlass es ermöglicht. 
In so manchen der flüchtig hingeworfenen Bemerkungen glauben wir dem schöpferischen Quell näher zu sein, spüren wir unmittelbarer den Hauch der großen Persönlichkeit, als in den ausgereiften Werken. Dort haben wir die ursprünglichen Einfälle und Eingebungen des Augenblicks vor uns, hier: was treuer Fleiss aus ihnen gemacht hat.

Nur aus seiner Entwicklung heraus kann man Kants System begreifen; und dem werdenden wie dem fertigen Gedankenbau wird man verständnisslos gegenüber stehn, solange man nicht das Geheimniss von Kants Individualität erfasst hat in ihrer ganzen Complicirtheit, mit ihren gegen einander strebenden Tendenzen, ihren Wünschen und Bedürfnissen, Denkmotiven und Denknothwendigkeiten. Alles das aber wird erst durch den Nachlass völlig erschlossen. Und darum musste er ganz veröffentlicht werden. Denn wer hătte auswählen sollen? Nach welchem Maasstab? Der Subjectivität wären alle Pforten geöffnet worden. Manches hätte man vielleicht achtlos bei Seite gelegt, aus dem spätere Generationen wichtigste Schlüsse ziehen. Denn anch das Kleinste, in seiner Vereinzelung ohne jeden Werth, kann und mag, im grossen Zusammenhang des Ganzen betrachtet, ungeahnte Bedeutung gewinnen.

Nur einer Beschränkung unterliegt leider das Gesagte. Das grosse unvollendete Manuscript, an dem Kant in den letzten Jahren seines Lebens arbeitete, und von dem R. Reicke in der Altpreassischen Monatsschrift (Bd. XIX-XXI, 1882-4) den grōsseren Theil veröffentlichte, war der Ausgabe nicht zugänglich und erscheint deshalb nicht in ihrem Rahmen.

\section{Anordnung des Stoffes.}

Um die wissenschaftliche Erforschung des handschriftlichen Nachlasses zu erleichtern, beziehungsweise überhaupt erst zu ermöglichen, musste eine sachliche Anordnung gewählt werden. Hätte man sich in der Eintheilung des Stoffes nach den Orten gerichtet, an denen sich die Handschriften jetzt befinden, so hätte man das Wirken des Zufalls zum obersten Dispositionsprincip erhoben. Auch die von Schubert vorgenommene Vertheilung der Losen Blätter der 